\title{
Lung abscess post bronchoscopy
}

\author{
Majdi AI Dliw MD, Haitham Mazek MD
}

\section{INTRODUCTION}

A lung abscess is an area of pulmonary parenchymal necrosis caused by bacterial infection. The most frequent cause is aspiration of anaerobic organisms from the mouth; other causes include necrotizing pneumonia and septic emboli. Lung abscesses can be classified as primary or secondary based upon the presence or absence of common associated conditions. Symptoms of lung abscess may include fever, malaise, cough with foul smelling sputum, hemoptysis, shortness of breath, and weight loss. We present a case of 55-year-old man who had a primary lung abscess after elective bronchoscopy.

\section{Case Presenttion}

A 55-year old man with no significant past medical history presented with six months of recurrent episodes of mild to moderate shortness of breath with no other pulmonary symptoms. His initial chest $x$-ray and CT scan of the chest showed an elevated left diaphragm and mild linear atelectasis in the left lower lobe. The patient was admitted for an elective bronchoscopy. At that time he denied any other symptoms, and his physical examination was within normal limits, including no dental or gingival disease. A flexible fiberoptic bronchoscopy with bronchoalveolar lavage was uneventful. The patient was observed for two hours and had a chest $\mathrm{x}$-ray after the procedure which showed no change. Four days later, the patient

Corresponding author: Haitham Mazek MD

Contact Information: Haitham.mazek@ttuhsc.edu DOI: 10.12746/swrccc2015.0309.124 came back with fever, chills, and hemoptysis for three days. On the physical examination, he was tachypneic and looked ill. His chest examination was significant for decreased air entry in the left middle/lower lobes and crackles in the left lower lobe. His lab studies were significant for leukocytosis. He was started on IV clindamycin and levofloxacin for a preliminary diagnosis of pneumonia. His chest $x$-ray revealed possible cavitation on the left lower lobe, and a CT of the chest confirmed that there was a $6.9 \mathrm{~cm} \times 3.7$ $\mathrm{cm}$ lung abscess present. The patient continued antibiotics, began to subjectively improve, and appeared less toxic; his WBCs returned to normal values. The patient underwent more work up to evaluate possible etiologies, including TB, HIV, histoplasmosis, and aspergillosis. All tests were negative. His blood cultures revealed no growth. The patient was discharged on IV antibiotics for six weeks, and a follow up CT of his chest two months later showed complete resolution of the abscess.
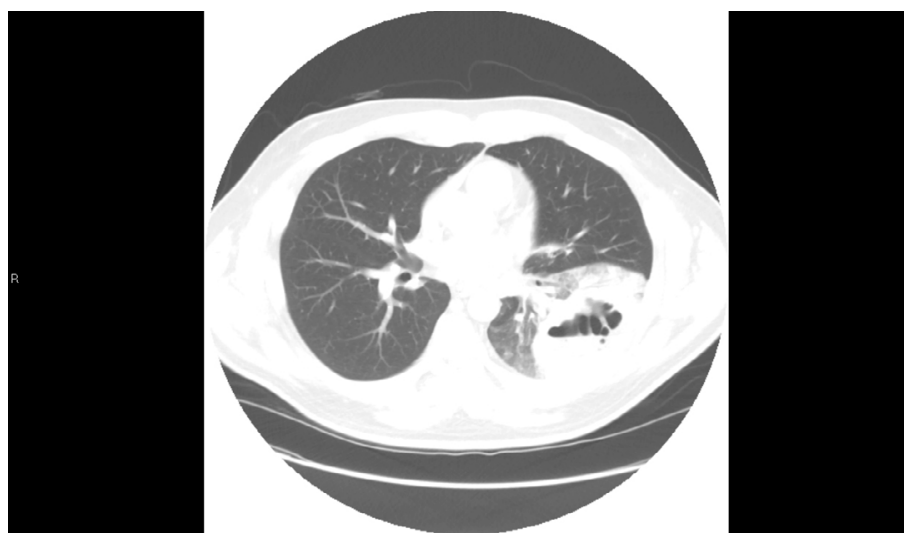

Figure : Computed tomography of the chest with lung window settings demonstrates a large cavitary infiltrate in the left lower lobe. 


\section{Discussion}

Bronchoscopy is a key diagnostic technique for many lung diseases and is generally considered safe. However, as with any invasive procedure, it can be associated with complications. The usual complications after an elective bronchoscopy tend to be minor, such as fever or transient parenchymal infiltrates. Serious complications, like pneumothorax or air embolism, can occur, but these are rare and occur mainly after transbronchial biopsies.' One study reported a mortality rate of $0.01 \%$ and a major complication rate of $0.08 \%$ in a series of 24,521 procedures, and another reported a $0.02 \%$ mortality rate and $0.3 \%$ major complication rate in a series of 48,000 cases. ${ }^{2}$

Lung abscesses can be classified into primary and secondary categories. Approximately $80 \%$ of lung abscesses are primary and develop as a result of primary infection of the lung. ${ }^{3}$ These commonly develop in necrotizing pneumonia following aspiration or in chronic pneumonia, such as pulmonary tuberculosis. Common causes for aspiration include alcoholic intoxication, seizures, stroke, drug overdose, and general anesthesia. Other causes for aspiration include dysphagia secondary to neurologic diseases, such as amyotrophic lateral sclerosis, Parkinson's disease, or stroke, or esophageal diseases, or following dental work. Secondary abscesses are caused by a preexisting condition; examples include airway obstruction from a neoplasm or foreign body, dissemination from an extrapulmonary site such as septic embolization from tricuspid valve endocarditis or suppurative phlebitis, a complication with intrathoracic surgery, or an immunosuppression from human immunodeficiency virus infection or immunosuppressive drugs. ${ }^{3}$

The incidence of lung abscess in an edentulous person is very low, and this association should raise the possibility of an airway obstruction, often caused by bronchogenic carcinoma. In some studies, almost $50 \%$ of lung abscesses in adults older than 50 years are associated with carcinoma of the lung, either because of infection behind an obstructing tumor or infection within the necrotic tumor itself.

Lung abscess accounts for 4.0 to 5.5 admis- sions per 10,000 hospital admissions each year in the USA. ${ }^{5}$ It is very infrequent after elective bronchoscopy. This case illustrates the need for vigilant precautionary measures, such as aseptic technique, during bronchoscopy to minimize the potential for infection and for post procedure follow-up.

\section{KEY WORDS}

lung abscess, bronchoscopy, complication

Author Affiliation: Majdi Al Dliw is a resident internal medicine at Rosalind Franklin University in Chicago, IL. Haitham Mazek is a resident in internal medicine at Texas Tech University Health Sciences Center in Lubbock, TX.

Received: $12 / 15 / 2014$

Accepted: 01/12/2015

Reviewers: Richard Winn MD, Tim Chen DO

Published electronically: 01/15/2015

Conflict of Interest Disclosures: none

\section{REFERENCES}

1. Pue CA, Pacht ER. Complications of fiberoptic bronchoscopy at a university hospital. Chest 1995; 107:430-2.

2. Suratt P, Smiddy J, Gruber B, Deaths and complications associated with fiberoptic bronchoscopy. Chest 1976; 69: 747-51.

3. Bennett JE, Dolin R, Blaser MJ. Bacterial lung abscesses. In Mandell, Douglas, and Bennett's Principles and Practice of Infectious Diseases. 8th ed. St Louis, MO: W B Saunders, 2014: 855-859.

4. Theodore PR, Jablons D. Thoracic wall, pleura, mediastinum, and lung. In Doherty G, Companies M. Current diagnosis and treatment: surgery. 13th ed. New York: McGraw Hill Professional, 2009: 305-358.

5. Chidi CC, Mendelsohn HJ. Lung abscess: a study of the results of treatment based on 90 consecutive cases. $J$ Thorac Cardiovasc Surg 1974; 68:168-172. 